\title{
Therapeutic principles in upper limb trauma
}

\author{
Andreea Grosu-Bularda', Florin-Vlad Hodea', Liviu-Petre Cojocaru', Alexandru Stoian', \\ Flavia-Francesca Lita', Serban Arghir Popescu ${ }^{1,2}$, Ioan Lascar ${ }^{1,2}$, Razvan Teodoreanu ${ }^{1,2}$ \\ ${ }^{1}$ Clinic of Plastic Surgery, Aesthetic and Reconstructive Microsurgery, Clinical Emergency Hospital, Bucharest, Romania \\ 2 "Carol Davila" University of Medicine and Pharmacy, Bucharest, Romania
}

\begin{abstract}
Upper limb trauma cases vary from simple to high energy impactful injuries, with different etiologies; situations which frequently require unique, demanding and challenging endeavors in order to obtain the most favorable outcome. Experience, good decision-making and knowledge of functional goals are mandatory in order to elaborate a therapeutic plan and execute it accordingly. Although cases differ in nature and prognosis, respecting a set of therapeutic principles whilst dealing with either simple or complex cases, will enhance patient outcome and give the surgeon the confidence to tackle any kind of upper limb trauma. After clearing out vital threat, the emergency surgery represents the first threshold in achieving and restoring normal function and biomechanics, mostly in young and labor active patients, with the mindset to salvage as much tissue as possible, with a thorough debridement and step-by-step approach to different types of tissues. Secondary surgery and reconstructive surgery can be planned timely, with prior discussion with both the therapist and the patient in order to enhance patient's upper limb function and aesthetic and ensure social reintegration.
\end{abstract}

Keywords: upper limb trauma, therapeutic principles, emergency surgery, surgical principles, therapeutic algorithm

\section{INTRODUCTION}

Upper limb trauma still presents to date, a demanding therapeutic challenge, due to the variability of the case with multiple external factors such as traumatic mechanism, duration of injury, anatomical site, previous functional status, time of ischemia, all-in-all generating cases ranging from simple to complex, such as the mutilated hand. The importance of prompt intervention in such cases is once again enhanced by the fact that these types of injuries occur in young and labor active patients, with both individual social and functional impact, as well as public health and economy impact given by the burden of upper limb sequelae [1,2].

Vital threat is not neglectable since complex injuries may include multiple structures and systems. Once the patient is cleared from a non-critical standpoint, without signs of critical condition, addressing the upper limb trauma becomes priority.
Some complex cases require immediate and thorough planification, a skilled and experienced team [3].

When planning for reconstruction, the surgeon must take into consideration patient factors such as general status, age, domination of hand, profession and socio-economic status. Pre-existing comorbidities significantly increase difficulty of the case, mainly cardiac affliction or vessel disease due to vasculitis, smoking status and diabetes mellitus. Other factors that also need to be considered are ischemia time, injury mechanism, wound contamination status, and history of limb trauma [4,5].

\section{THERAPEUTIC PRINCIPLES}

\section{General principles}

Life always is the utmost priority and any attempts over saving a limb with vital risk should be 
discarded. Adherence to the Advanced Trauma Life Support (ATLS) always precedes surgical intervention, with ample and thorough evaluation, since patients suffering from upper limb trauma may possess other life-threatening conditions that need addressed promptly and first-hand treatment [6-8].

Resuscitation is a priority over the extremity trauma; however, one should not completely neglect the affected limb while life support is instated. Ischemia evaluation, reduction of gross deformity and splinting are not time consuming and can be done initially before attempting any major surgery [9].

\section{Surgical principles}

\section{Treatment goals}

Preserving as much tissue as possible is key, implicitly preserving function whenever possible. If not possible, a surgical plan to reconstruct and restore function should be kept in mind, as well as taking into consideration multiple back-up options should the initial attempt fail [10].

Preservation of function of the upper extremity comprises of two major goals, the first being either maintaining or reestablishing normal sensibility, the latter being restoring normal biomechanics of the injured limb. Minimal hand function is obtained with an opposable thumb and another stable unit. If possible, all other function will be reconstructed in the following order: thumb-index pinch, key pinch, then hook and chuck grips, span and power grasps and lastly finesse, precision movements [11].

\section{Emergency surgery principles}

A step-by-step protocol should be employed in an emergency setting. Hemorrhage should then be addressed by either direct pressure with emphasis on avoiding clamping without a clear view of the vessel bleeding source; If ligatures are performed, they should be placed as distal as possible on the vessel ends. This measure aims to avoid further loss of length leading to vessel gap, as a useful measure when attempting end-to-end vessel anastomosis. Tourniquets can be employed to aid massive bleeding control, with release at every two hours [12].

Gross skeletal deformity should be reduced, with splinting, whenever possible. Tetanus prophylaxis and empiric antibiotics should be administered. If the limb is ischemic, a temporary vascular shunt should be used, with cooling of the affected part with a protective barrier to avoid frostbite. Lastly, any skin, or tissular bridges should be left intact in this stage [13].

\section{Reconstructive surgery principles}

Debridement, with abundant irrigation, is the first step towards an efficient intervention. Cul- tures, should be taken, although the wound may look clean, having a good prediction of later-infected wounds, guiding the antibiotic therapy according to antibiogram. Wound excision is mandatory, followed by initial surgical scrubbing with gravity assisted lavage. One should always begin with a tourniquet, with release for evaluation of tissue viability and reinflation if further debridement is needed [9]. Figure 1 presents a case of a hydraulic press injury with compartment syndrome.
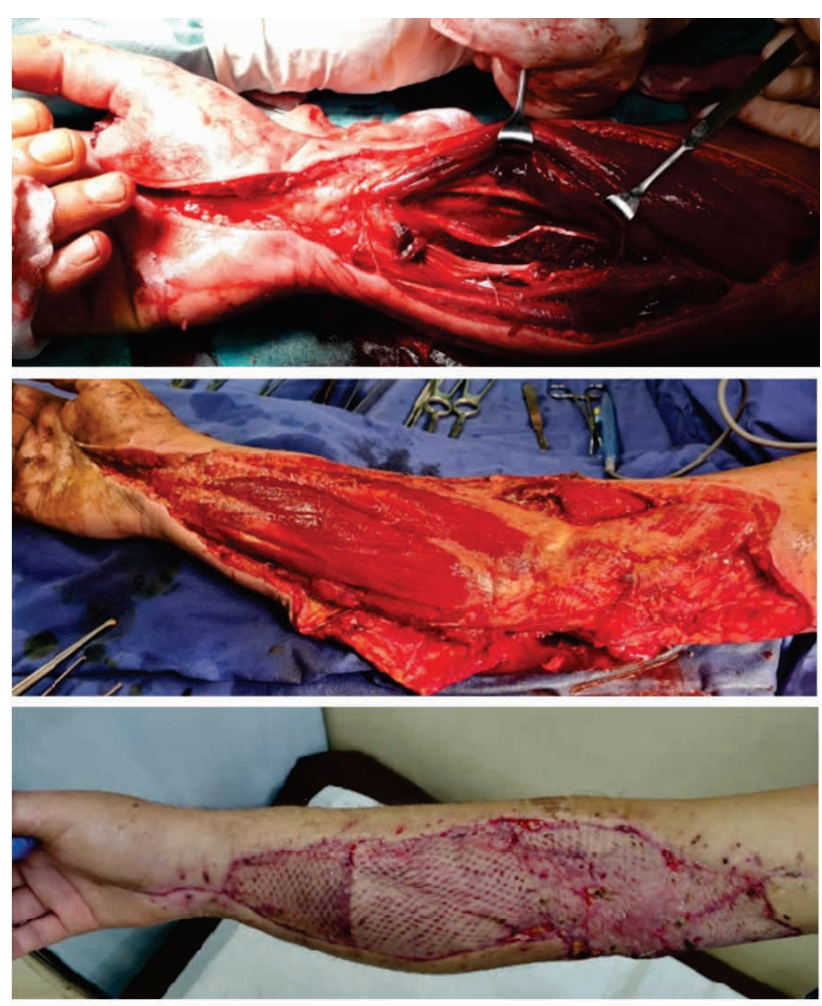

FIGURE 1. Crush injury (industry rolling machine) initially with compartment syndrome and devitalized profound musculature - before debridement, followed by fasciotomy and excision of devitalized tissues - after debridement, and lastly 28 days post-coverage with split thickness skin graft

Aggressive debridement should be done until vascularized tissue is noticed. Skin and subcutaneous tissues should be trimmed to bleeding edges. The muscles are debrided until bright red color is spotted and both mechanical and electrical stimulation is positive. Small bony fragments, devoid of vascularization, should be extracted or resected. Critical structures that should be saved are arteries, nerves and tendons and should be tagged $[11,14]$.

An experienced surgeon should decide about replantation, amputation, partial amputation, limb salvage or reconstruction. Necessity amputation is sometimes inevitable as part of the debridement process in emergency surgery. Indication for emergency amputation is taken into consideration should the trauma involve open fractures with large 
soft-tissue defects, prolonged warm ischemia with muscle involvement, unstable biological status, associated organ trauma, vital risk with longer periods of anesthesia, as tentative limb preservation attempt may preclude vital prognosis [15]. Figure 2 presents a case of a firecracker explosion. As much tissue as possible was salvaged. Figure 3 presents two separate cases of hand replantation and thumb replantation.

The upper limb amputation poses a higher social, aesthetic and functional impact than lower limb amputation, with higher rates of prosthetic rejection, needing complex rehabilitative protocols. Functional recovery is faster in case of lower limb amputation, with faster social reintegration, compared to upper extremity amputation $[9,16,17]$.

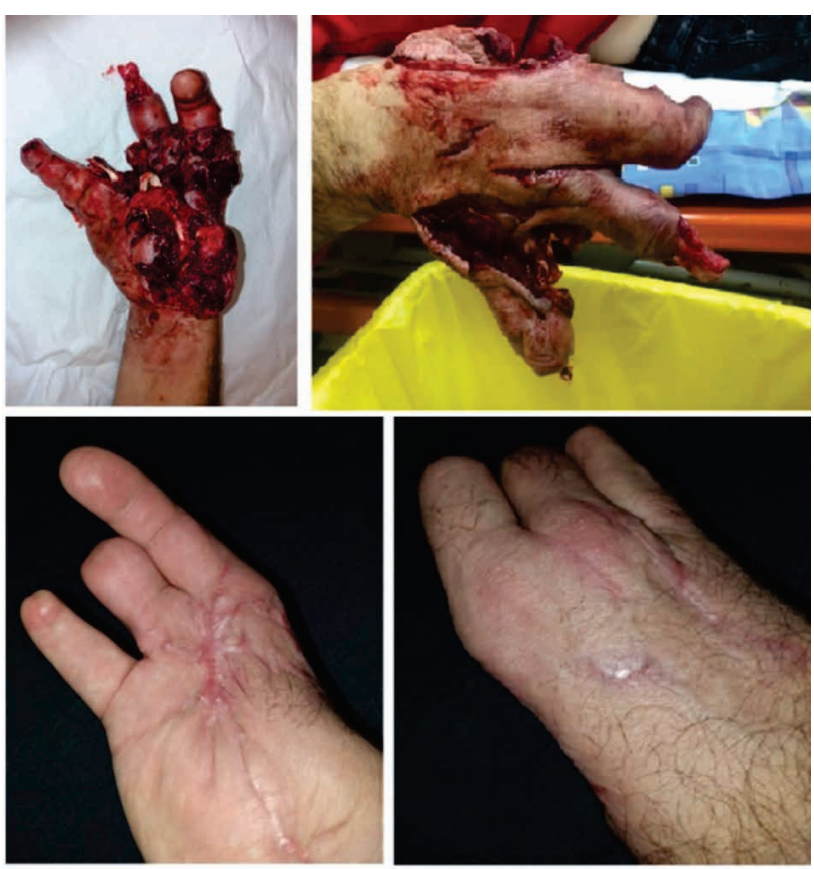

FIGURE 2. Firecracker explosion - presentation and follow-up
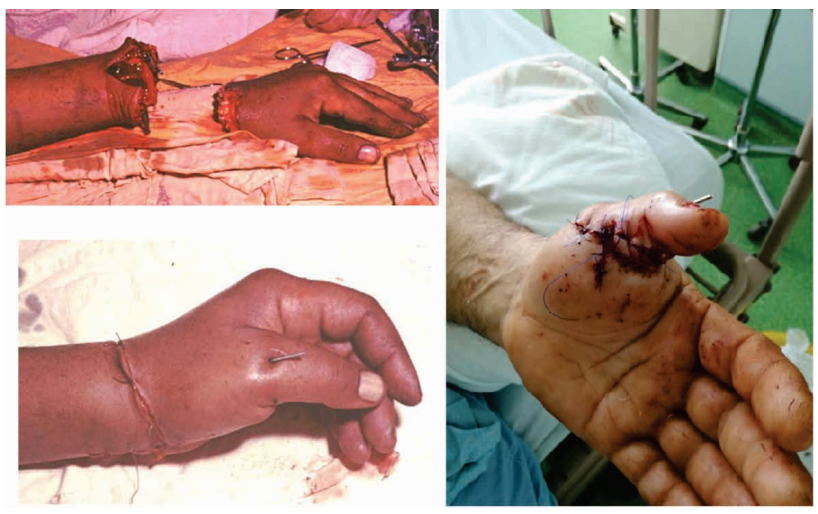

FIGURE 3. Cases of replantation

Vascularized bone should be saved for reintegration, as well as vascularized soft tissues in order to attempt coverage or closure. Salvage of spare parts should be attempted whenever possible, usable lat- er in primary reconstruction. Lastly, open contaminated wounds should be left exposed, with needed sequential debridement to be performed in the following day.

When it comes to skeletal defects, one should decide between shortening, primary bone grafting, or placement of an antibiotic spacer with delayed bone grafting.

External fixators, internal fixation with screws, plates, K-wires or cerclage can be performed depending on the severity of bone trauma. From a reconstructive and rehabilitation standpoint of view, stable and definitive internal fixation is always preferable to external fixation. On the other hand, external fixation is an excellent temporary solution to heavily contaminated wounds $[9,15,18]$. Figure 4 shows a metacarpal fracture, that upon debridement was left with a significant gap. Iliac crest bone was harvested and used to fill in the defect.
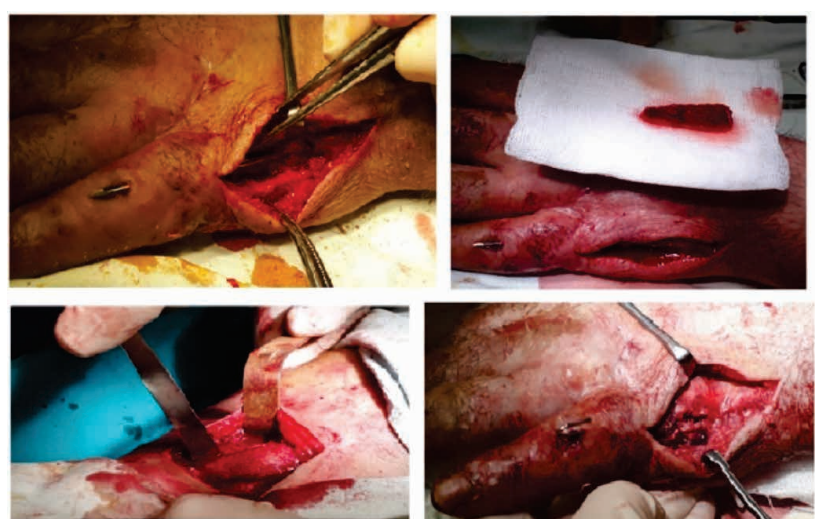

FIGURE 4. Fifth metacarpal defect reconstructed using iliac crest bone graft

Tendon repair begins with debridement of its crushed muscle in order to prevent contractures. The surgeon should always identify both ends of the musculotendinous unit. Should the muscle be damaged, it should be debrided accordingly as aforementioned, and distal tendinous ends may require sometimes repair to other available units proximally, with emphasis on synergistic actions [19,20].

Core locking sutures for flexor tendons should employ a multiple stranded technique, in addition to a fine epitendinous suture. Both flexor digitorum profundus and digitorum superficialis should be repaired, whenever possible, the exception being when gliding is impaired, in which case the flexor digitorum profundus alone should be repaired. A2 and A4 pulley repair or reconstruction should be performed, either primarily or by using tendon grafts. Primary tenodesis/tendon transfer should be considered. For late reconstruction, tendon grafting, tendon transfer, tenolysis and functional free muscle transfer may be needed [21,22]. 
Vascular repair/reconstruction should be performed after skeletal and tendon repair unless the limb distal to the traumatic level is ischemic. In such case a temporary shunt can be employed. Before trimming the vessel ends until healthy, uninjured tissue is observed, a Fogarty catheter should be used to de-clot the damaged vessel. The vessel ends should be irrigated with heparin solution and then clamped. The repair itself should be performed under the microscope, outside of injury site is possible. In case of inadequate length after trauma or debridement itself, ligation of tributaries, joint flexion, or usage of a vein graft harvested outside injury zone are all reliable measures. Repair of all major vessels should be considered, e.g., both radial and ulnar arteries at the level of the forearm, whenever possible. Venous reconstruction is required only if all or nearly all venous outflow is deficient or absent. Medical leeches can be used in case of venous congestion [21,23].

Nerve repair is the next step after skeletal, tendon and vascular repair and should be performed under the microscope, with proper alignment. This represents the last step before soft tissues coverage. Even though nerve grafts and nerve transfers gain more and more popularity lately, one should take into consideration that primary end-to-end nerve repair represents the therapeutic gold standard [24].

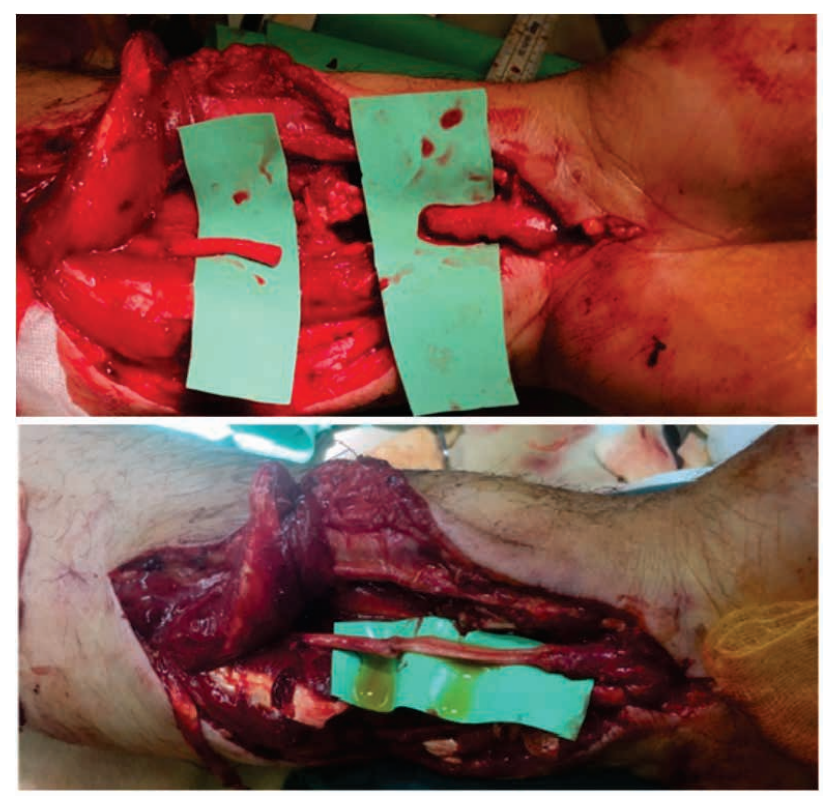

FIGURE 5. Median nerve gap, followed by repair using sural graft

If the nerve is concussed, it should be left alone, avoiding further iatrogenic damage to it. Should it be severed, the crushed ends must be trimmed to healthy nerve fascicles, followed by epi/peri-neural repair in a tension-free manner, respecting the fascicular and surface anatomy for proper alignment.
Should a short gap be present, flexion of the joint or usage of a conduit are useful measures, but for larger gaps, utilization of nerve grafts is sometimes the best decision. If not nerve is not repaired initially, the nerve ends should be anchored to facilitate nerve repair during the next intervention [24,25]. Figure 5 reveals the gap left after median nerve ends trimming until healthy fascicles were found. Sural nerve harvest was decided for nerve repair.

\section{Soft tissues coverage}

As aforementioned, sequential debridement may be needed for heavily contaminated or unstable wounds. Therefore, one should always delay definite coverage until stable wound conditions are met. Well vascularized, thin and supple tissues are required to cover joint and tendons. Volar pressure-bearing surfaces should be covered with sensate and minimal shearing tissues. Wound should be kept moist until definitive coverage. Negative pressure wound therapy is always a good measure that can buy time, the condition being absence of infection or active bleeding. Complex wounds with exposed "white structures" (tendon, nerve, bone or ligament/joint) should always be covered. Traumatically created flaps should not be stretched to their original position since the mechanical tension applied to it may cause necrosis of poorly perfused areas [26].

The aims of soft tissue coverage in upper extremity trauma are limb salvage, allowing healing of the underlying injuries, simultaneous reconstruction of composite defects and enabling recovery and mobility as fast as possible, with function restoring the main goal, as much as possible [27].

One should always keep in mind the concept of "reconstructive ladder", when planning soft tissue reconstruction. Complexity increases from healing by secondary intention, primary closure, skin grafting, local flaps, regional flaps and lastly usage of free flaps. Negative pressure wound therapy is a great tool that gives time to develop a plan, while maintaining the stability of the wound [28].

The surgeon should always keep at least one back-up plan to fall upon in case of failure of each of the aforementioned layers of the reconstructive ladder.

Secondary procedures are often necessary for optimization of upper limb function and should be planned in a staged manner. Figure 6 reveals a crush injury with an unstable wound - extensive devitalization, extensor tendons avulsion, scaphoid dislocation, bone exposure and skin necrosis. Initially, debridement was performed until healthy tissues were found. Second stage procedure was performed with pedicled flap for coverage of dorsum 

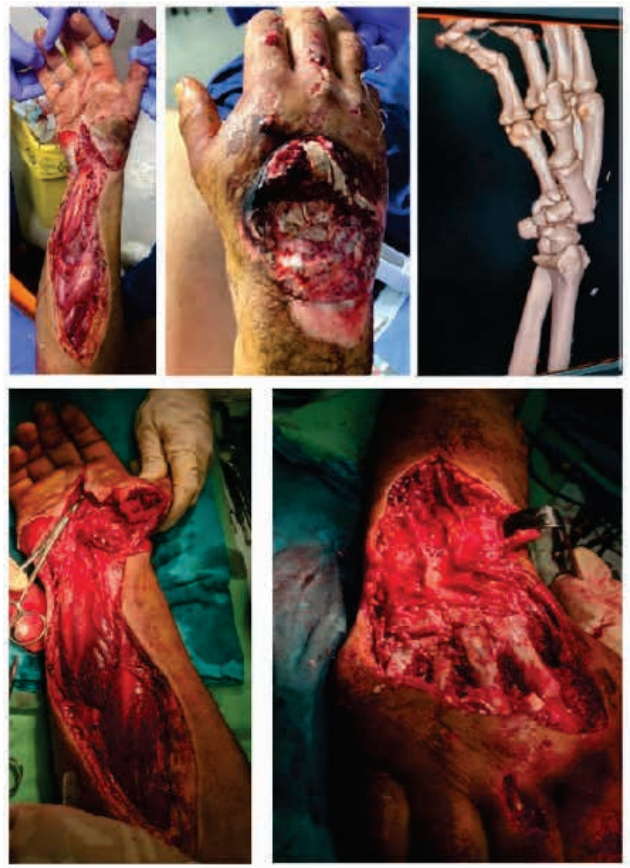

FIGURE 6. Crush injury with an unstable wound
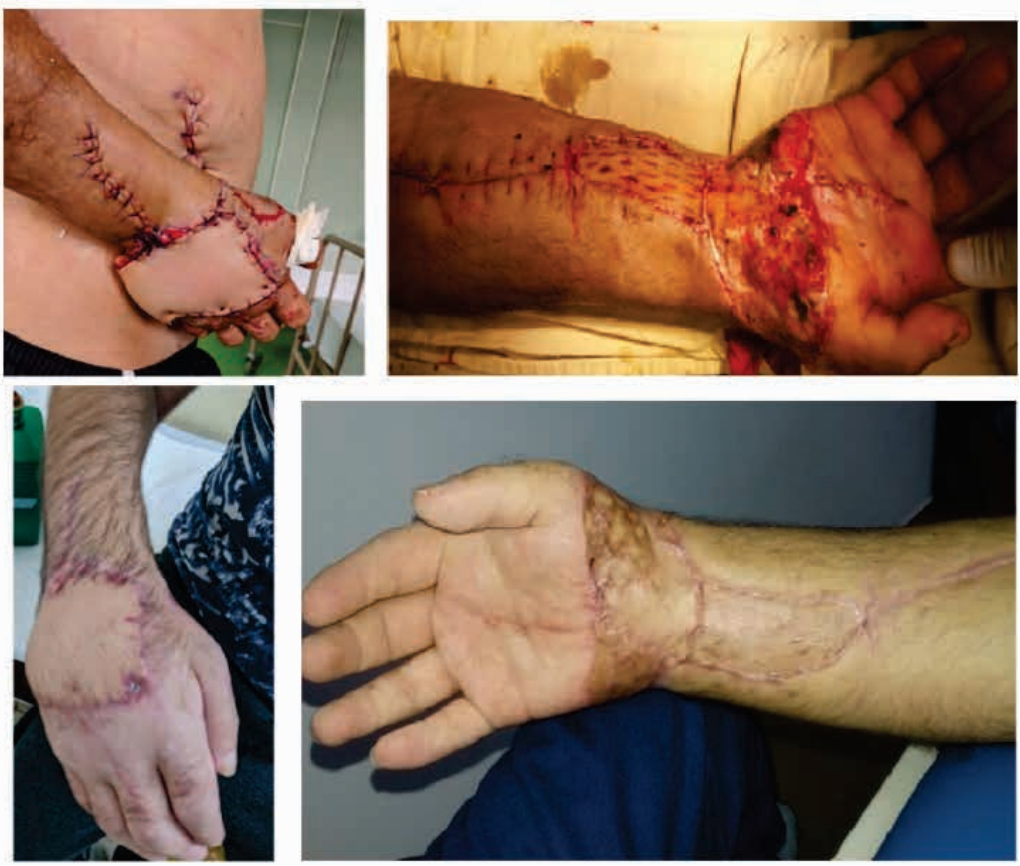

of the hand and skin graft for the volar aspect of the wrist.

Firstly, there are interventions that require segment immobilization such as bone grafting, corrective osteotomies, joint reconstruction, nerve grafting, tendon transfers, functional muscle transfer, soft tissue reconstruction, toe transfer. Soft tissue reconstruction can be seen in Secondary procedures that require mobilization are tenolysis, capsulotomy, contracture releases, performed in delayed fashion rather than the aforementioned procedures.

\section{Rehabilitation principles}

A collaboration with hand therapist, with adherence to the rehabilitation protocol is essential in order to gain an adequate recovery. Appropriate splinting, either static or dynamic, should be applied to diminish as much as possible future contractures. Patients with extensive upper limb trauma often present functional impairment and chronic sequelae, requiring long-term follow up and need strict adherence institutional rehabilitation programs to be easily reintegrated socially and re-enter the labor active population [29].

Early motion, whenever possible should be started, improving and optimizing long-term function. Edema is managed, desensitization should be initiated. Lastly, the psychological status of the patient should be addressed based on socio-economic background, origins and level of education in establish and achieve a pre-established set of goals $[9,30]$.

\section{DRAWBACKS}

The level of rehabilitation care in upper limb trauma is not uniformly worldwide distributed, being dependent on infrastructure, dedicated hand surgery centers and dedicated hand therapy centers. Developing countries present variable degrees of addressability to rehabilitation programs, given patient's origins, socioeconomical and psychological status.

Severe injuries in patients with poor social status, with rural origins, have low accessibility and low addressability to rehabilitation therapies and corrective/ secondary procedures. Prostheses are not affordable for the large majority.

One solution would be promotion of reconstructive surgery educational programs, increasing patient addressability, with long term follow-up with realistic goals, objective evaluation of functional results using scoring systems. All efforts are made to perform optimal surgical reconstruction and provide the best patient care possible. At the current moment, in our clinical setting, given the demographical status, for our patients, a reconstructed upper limb with sensate function is better than a prosthesis.

\section{PERSPECTIVES}

Adherence to recent developments in reconstructive field should be encouraged, with an always updating surgeon to the latest discoveries and being up to date with newfound technology. Vascu- 
larized composite allografts, emphasizing on bilateral upper limb transplant is a viable solution, with an impressive reconstructive potential. Ongoing and current literature data suggest less toxic and aggressive immunosuppressant protocols can be adopted, which can propel this field into an excellent solution for a specific category of patients. Lastly, targeted muscle reinnervation also may be an excellent choice, showing impressive functional outcome.

\section{CONCLUSIONS}

Even though, upper extremity injuries can be challenging, time-saving decisions can be easily and immediately taken, in order to generate favorable outcomes, should the surgeon keep a therapeutic algorithm in mind. Experience on the other hand represents the other facet needed by the upper limb

\section{$\overline{\text { REFERENCES }}$}

1. Lee IJ, Cha B, Park DH, Hahn HM. Role of plastic surgeons in the trauma center: national level I trauma center startup experience in South Korea. Medicine (Baltimore). 2021 Feb 5;100(5):e24357.

2. Singhal M, Naalla R, Dave A, Moumita De, Gupta D, Chauhan S. The role of plastic and reconstructive surgeon in trauma care: Perspectives from a Level 1 trauma centre in India. Indian J Plast Surg. 2018 May-Aug;51(2):170-176.

3. Win TS, Henderson J. Management of traumatic amputations of the upper limb. BMJ. 2014 Feb 10;348:g255.

4. Neumeister M, Hegge T, Amalfi A, Sauerbier M. The reconstruction of the mutilated hand. Semin Plast Surg. 2010 Feb;24(1):77-102.

5. Ng ZY, Askari M, Chim H. Approach to complex upper extremity injury: an algorithm. Semin Plast Surg. 2015 Feb;29(1):5-9.

6. Jordan DJ, Malahias M, Khan W, Hindocha S. The ortho-plastic approach to soft tissue management in trauma. Open Orthop J. 2014 Oct 31;8:399-408.

7. van Maarseveen OEC, Ham WHW, van de Ven NLM, Saris TFF, Leenen $\mathrm{LPH}$. Effects of the application of a checklist during trauma resuscitations on ATLS adherence, team performance, and patientrelated outcomes: a systematic review. Eur J Trauma Emerg Surg. 2020 Feb;46(1):65-72.

8. Bernstein ML, Chung KC. Early management of the mangled upper extremity. Injury. 2007 Dec;38 Suppl 5:S3-7.

9. Chang J, Neligan P. Volume 6: Hand and Upper Limb. Plastic Surgery, 4th Edition. Elsevier, 2017.

10. Bumbasirevic M, Stevanovic M, Lesic A, Atkinson HD. Current management of the mangled upper extremity. Int Orthop. 2012 Nov;36(11):2189-95.

11. del Piñal F. Severe mutilating injuries to the hand: guidelines for organizing the chaos. J Plast Reconstr Aesthet Surg. 2007;60(7):816-27.

12. Kragh JF Jr, Walters TJ, Baer DG, Fox CJ, Wade CE, Salinas J, Holcomb JB. Survival with emergency tourniquet use to stop bleeding in major limb trauma. Ann Surg. 2009 Jan;249(1):1-7.

13. Hornez E, Boddaert G, Ngabou UD, Aguir S, Baudoin Y, Mocellin N, Bonnet S. Temporary vascular shunt for damage control of extremity vascular injury: A toolbox for trauma surgeons. J Visc Surg. 2015 Dec;152(6):363-8.

14. Scheker LR, Ahmed O. Radical debridement, free flap coverage, and immediate reconstruction of the upper extremity. Hand Clin. 2007 Feb;23(1):23-36. trauma surgeon, mandatory to tackle cases ranging from simple to complex. If the surgeon adheres to a basic step-by-step set of principles at every step of the chain of events while treating upper limb trauma cases, short-term outcome and long-term prognosis improves, all the while maximizing efficiency throughout the process.

Careful and complete evaluation of the patient and injury is mandatory. A well formulated and comprehensive plan with back-up solutions should be employed, adapted to the patient's needs, background, and possibilities, with realistic expectations. Upper limb trauma frequently involves sub-sequential surgical interventions in order to reconstruct and restore function and appearance. Comprehensive rehabilitation of the upper limb is crucial with strict adherence by the patient. Continuous education is needed to ensure ever-upgrading standards of care by the medical professionals.

\section{Conflict of interest: none declared Financial support: none declared}

15. Langer V. Management of major limb injuries. ScientificWorldJournal. 2014 Jan 5;2014:640430.

16. Desteli EE, Imren Y, Erdoğan M, Sarısoy G, Coşgun S. Comparison of upper limb amputees and lower limb amputees: a psychosocial perspective. Eur J Trauma Emerg Surg. 2014 Dec;40(6):735-9.

17. Marchessault JA, McKay PL, Hammert WC. Management of upper limb amputations. J Hand Surg Am. 2011 Oct;36(10):1718-26.

18. Alphonsus CK. Principles in the management of a mangled hand. Indian J Plast Surg. 2011 May;44(2):219-26.

19. Bumbasirevic M, Stevanovic M, Lesic A, Atkinson HD. Current management of the mangled upper extremity. Int Orthop. 2012 Nov;36(11):2189-95.

20. Chattopadhyay A, McGoldrick R, Umansky E, Chang J. Principles of tendon reconstruction following complex trauma of the upper limb. Semin Plast Surg. 2015 Feb;29(1):30-9.

21. Wolfe SW, Pederson WC, Kozin SH, Cohen MS. Green's Operative Hand Surgery $7^{\text {th }}$ ed. Elsevier, 2016.

22. Dy CJ, Daluiski A. Flexor pulley reconstruction. Hand Clin. 2013 May;29(2):235-42.

23. Feliciano DV. Management of peripheral arterial injury. Curr Opin Crit Care. 2010 Dec;16(6):602-8.

24. Moore AM, Wagner IJ, Fox IK. Principles of nerve repair in complex wounds of the upper extremity. Semin Plast Surg. 2015 Feb;29(1):40-7.

25. Griffin MF, Malahias M, Hindocha S, Khan WS. Peripheral nerve injury: principles for repair and regeneration. Open Orthop J. 2014 Jun 27;8:199-203.

26. Miller EA, Friedrich J. Soft Tissue Coverage of the Hand and Upper Extremity: The Reconstructive Elevator. J Hand Surg Am. 2016 Jul;41(7):782-92.

27. Ng ZY, Salgado CJ, Moran SL, Chim H. Soft tissue coverage of the mangled upper extremity. Semin Plast Surg. 2015 Feb;29(1):48-54.

28. Levin LS. The reconstructive ladder. An orthoplastic approach. Orthop Clin North Am. 1993 Jul;24(3):393-409.

29. Novak CB, von der Heyde RL. Rehabilitation of the upper extremity following nerve and tendon reconstruction: when and how. Semin Plast Surg. 2015 Feb;29(1):73-80.

30. Miller LK, Jerosch-Herold C, Shepstone L. Effectiveness of edema management techniques for subacute hand edema: A systematic review. J Hand Ther. 2017 Oct-Dec;30(4):432-446. 